\title{
ACTUARIAL REMARKS ON PLANNING AND CONTROLLING IN REINSURANCE
}

\author{
By ERwin Straub
}

Zurich

\section{SKETCH OF THE PLANNING AND CONTROLLING PROCESS IN A (RE)INSURANCE COMPANY}

Planning is, or ought to be, an ever-developing process in which virtually each member of the company has to be involved. Planning without controlling, i.e., without feedback, planning on its own, is useless.

In the first part of the present note, general aspects of planning are briefly described inasfar as they are relevant to possible treatment by actuarial methods.

\section{1. "Hardware" and "Software" of Planning}

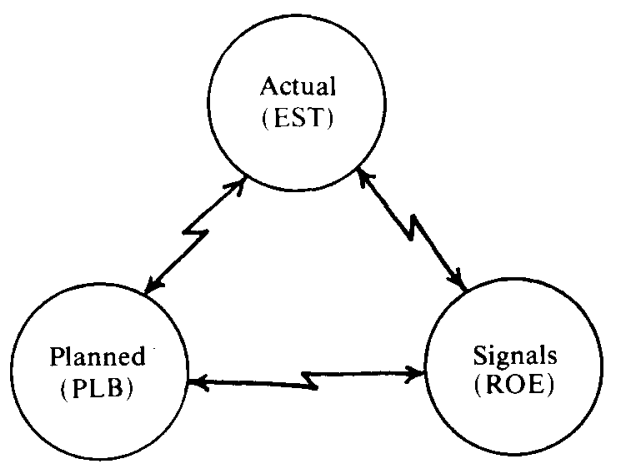

The circles in the above figure represent what could be called the hardware of planning consisting of three sets of figures, namely

- Actual figures describing the most recent reality. Most of these figures are part of what is usually called the Earnings Statement (EST).

- Forecasts for the near future, say the next three years. Most of these figures are contained in the Planning Budget (PLB).

- Control figures or signals in the sense of a "bread-line" expressing, for example, how much the company should earn as a minimum in order to remain selffinancing. Such figures are calculated on the basis of so-called Return on Equity considerations and they are found in a corresponding ROE-document.

By software we mean everything done with the hardware described above, i.e., primarily the comparison of figures from different domains of the hardware on 
different levels within the organisation of the company, the analysis, for example, of deviations between actual and planned, the conclusions to be taken from such analyses and the planning of new actions as a consequence.

In the above graph, circles symbolize the hardware and arrows indicate the software.

Also non-numeric planning instruments (e.g., project planning, action plans, assessment and decisions) are considered to be part of the planning software.

\subsection{Earnings Statement and Planning Budget}

The Earnings Statement and the Planning Budget are the two most important numerical management accounting tools for planning and controlling a reinsurance group and/or company and/or its various profit centers. The structure of both is the same and can be sketched as follows:

Operating Result Reinsurance (Non Life) consisting of

Premiums

Underwriting Result gross

Retrocessions

Change in IBNR

Management Expenses

Standard Investment Income on technical reserves

Adding these components together--each of them to be taken with its correct positive or negative sign-yields the operating result (before tax) of the reinsurance production unit in question (e.g., a marketing department, a geographic area, a specific product of the whole company).

Looking at this operating result over a number of years we observe that it is affected by two kinds of fluctuation, namely

- cyclical variations due to pulsation of the markets and

- random variations due to the occurrence (or non-occurrence) of large claims.

While it is of vital importance to judge (past and future) cyclical variations as realistically as possible in order to be able to react both adequately and in time, random variations are of quite a different nature and therefore require a completely different statistical treatment. Such a treatment is described in Section 1.3 below.

Clearly it is not at all easy to distinguish clearly between cyclical and random fluctuations in practice because the total fluctuation of the operating result is a mixture of both. Random fluctuations appear as a kind of noise or disturbance which makes it difficult to quantify the underlying cyclical changes and trends.

\subsection{The Cat Fund Concept}

Basically random fluctuations of operating (or underwriting) results can be smoothed either by external reinsurance (or retrocession) or internally by some 
kind of catastrophe and large claims fund. In what follows we shall mostly speak in terms of such internal arrangements, although the inherent ideas are the same both for an actual external cover and an internal arrangement which exists only on paper.

Looking at a reinśurance company from an organizational point of view, it could be conceived as a sort of profit center hierarchy.

Example:

$$
\text { PC 1 top level }=\text { level } 1=\text { whole company }
$$

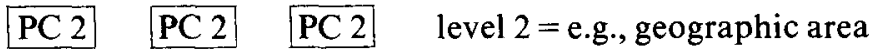

PC 3 level 3=e.g., line of business within area

We assume for the following that systematic planning and controlling is institutionalised in the company; in the present context this would mean among other things that

- an Earnings Statement (every year) and

- a Planning Budget (say every three years)

are produced for each profit center in the above figure. Clearly the Earnings Statement of a PC 2 is the sum of the Earnings Statements of all its PC 3s (and similarly for PC 1 of all its $\mathrm{PC} 2 \mathrm{~s}$ ). This sounds trivial but is nonetheless relevant in practice when there are different currencies, for example.

Whether a claim is considered as large or small depends on the size and structure of the portfolio under consideration. As a consequence of this, any mechanism designed to eliminate the "noise" must be much more efficient on the lower level profit centers (with the smaller volumes) than on top level.

This goal can be achieved in practice by working with two instruments, namely a Catastrophe Protection or Cat Cover acting on level 1 and a so-called Large Claim Compensator acting on the profit centers of level 3 defined as follows:

(i) The Cat Cover is an excess of loss arrangement where the retrocessionaire pays the excess of each claim which for the account of PC 1 exceeds a priority $P$ (say $£ 1$ million), however not more than a certain cover amount $C$ (say $£^{\prime} 19$ million, depending on the top catastrophe exposure). This Cat Cover is either placed with external retrocessionaires or consists of a company-internal catastrophe excess of loss arrangement, a Cat Fund. In practice the whole Catastrophe Protection is often a combination of both.

(ii) The Large Claim Compensator, an internal excess of loss mechanism, is usually a lower layer to the Cat Cover, paying the excess of every claim which for the account of any PC 3 is larger than $p$ (say $£ 100000$ ) up to where the Cat Fund comes in.

Note that the point "where the Cat Cover comes in" can vary from case to case: If a large claim hits one single PC 3 only with a gross amount of $£ 3$ million, then 
the Cat Cover pays $£ 2$ million to PC 1 as well as to the said PC 3 , and thus the Large Claim Compensator is left with an obligation of $£ 900000$ to that same PC 3. If, however, a $£ 3$ million claim hits two PCs of level 3, say $A$ with $£ 600000$ and $B$ with $£ 2.4$ million then again the Cat Fund's payment to the top PC 1 is two million, of which $£ 400000$ to the $A$-PC 3 and $£ 1.6$ million to $B$ so that the underlying Large Claim layer is left with payments of $£ 100000$ and $£ 700000$ to $A$ and $B$ respectively.

So the structure of the Earnings Statement and the Planning Budget (of any profit center from level 1 down to 3 ) sketched in Section 1.7 is incomplete. The full structure is rather:

\author{
Premiums \\ Gross underwriting result \\ Retrocessions \\ Change in IBNR \\ Contributions to \\ Recuperations from \\ Contributions to \\ Recuperations from \\ Cat Cover \\ Management Expenses \\ Standard Investment Income on technical reserves
}

\title{
1.4. Return on Equity Considerations
}

The purpose of calculating ROE minimum control figures is to give a quantitative answer to the following two basic questions:

(i) How much equity does a company need in order to run its business? $(\mathrm{ER}=$ equity required)

(ii) How much should the company earn as a minimum on its ER? (ROE minimum $=$ minimal return on equity)

Both ER and ROE are control figures which immediately lead to further questions, such as:

(i) How does the actual (or planned) equity of the company compare with its ER?

(ii) How does the actual (or planned) overall operating result of the company compare with its ROE minimum?

The basic idea underlying the ROE calculations is the criterion that the Group company should be self-financing. This may in some cases be a very severe criterion since after all a company can only do as well as the marketplace will allow. Nevertheless, if the minimum ROE is higher than the actual result, this is, to say the least, an important piece of information to the General Management. 


\section{ACTUARIAL CONTRIBUTIONS TO PLANNING}

Now the question is "What can an actuary do in the corporate planning department of an insurance or reinsurance company?" Below are indicated some possible answers to a few specific planning problems.

\subsection{How to Fix Cover Amounts and Priorities in the Cat Fund Arrangement}

The amount of coverage under the Cat Protection is dictated by the needs, i.e., the exposures written by the company. As a rule of thumb the upper point of the catastrophe cover is in the region of $10 \%$ of the company's GNPI (underlying gross net premium income) or less.

What is a reasonable priority $P$ ?

As a guideline for fixing $P$ under the Cat Fund we can argue that if the average operating result is $x \%$ of the GNPI then $P$ must be much below that since, if it were of the same order of size, then one single large claim after deduction of recuperation from the Cat Cover would already destroy the entire operating result. As the latter is perhaps in the region of $1 \%$ to $5 \%$, the priority $P$ should be some ten times less, say $0.3 \%$ of GNPI. The same reasoning is used for fixing the priorities $p$ under the Large Claim Compensator.

\subsection{Assessing the Cat Fund's Size and the Yearly Contribution to the Fund}

A practicable rule of thumb is to say that the fund should be able to pay a secular catastrophe claim, i.e., a catastrophe which happens in all likelihood only once in a century (such as the 1923 Tokyo earthquake or the Betsy windstorm in 1965, but also an imaginable secular man-made catastrophe which could hit the portfolio).

Another pragmatic approach is reflected in a rule of thumb of the type

$$
\text { reserve }=\frac{\text { fluctuation }}{\text { premium loading } \times \text { risk willingness }},
$$

the basic idea of which can be formulated as follows:

On the one hand the Cat Fund size (=reserve) must vary directly with the potential fluctuations of its claim load, while on the other hand it can be lower for higher loadings in the contribution to the fund and the more one is prepared to accept that the fund may be exhausted (=risk willingness). Intuitively this sort of connection between the above four items is pretty obvious; no actuarial model is needed to see this.

When it comes to quantifying things like "fluctuation" or "risk willingness" we cannot, however, do without a risk theoretical model. Considering the most simple actuarial model of insurance being a reservoir with steadily inflowing premiums and stochastically outflowing claims and putting equality in Cramér's 
inequality one obtains the following general formula of which the above mentioned rule of thumb is but a special case, namely

$$
\frac{E[Z]}{U} \cdot v[Z] \cdot\left(-\frac{\ln \varepsilon}{2}\right)=\frac{E[\tilde{Y}] / E[Z]}{\operatorname{Var}[\tilde{Z}] / \operatorname{Var}[Z]}
$$

of which a sketch of a proof is given under 2.6 below and where $U$ is the reserve, $Z$ stands for the yearly gross claims load of any insurance company or portfolio under consideration, $E[Z]$ its expected value i.e., the pure gross risk premium, $v[Z]=\operatorname{Var}[Z] / E^{2}[Z]$ which is the square of the coefficient of variation of $Z$, i.e., a measure for fluctuation, $\varepsilon$ is the tolerated probability of ruin, $\tilde{Y}$ and $\tilde{Z}$ are the net yearly result and claims load tespectively under some arbitrary reinsurance cover.

Putting $\tilde{Y}=Y$ and $\tilde{Z}=Z$ in the above formula yields

$$
\frac{U}{E[Z]}=\frac{v[Z]}{(E[Y] / E[Z])(-2 / \ln \varepsilon)}
$$

or in other words

$$
\text { reserve }=\frac{\text { fluctuation }}{\text { premium loading } \times \text { risk willingness }}
$$

with

$$
\text { "reserve" }=\frac{U}{E[Z]}
$$

i.e., the initial reserve $U$ is to be expressed as a multiple of $E[Z]$

$$
\text { "premium loading" }=\frac{E[Y]}{E[Z]}=\text { profit margin }
$$

since if $Y=P-Z$ with premiums $P=(1+\delta) E[Z]$ then $E[Y] / E[Z]=\delta$

$$
\text { "risk willingness" }=\frac{-2}{\ln \varepsilon}
$$

where $\varepsilon$ denotes the probability with which we allow the fund to be exhausted at some future time.

\subsection{Breakdown of Overall Risk Capital on Subportfolios}

Risk capital (sometimes also called contingency or fluctuation reserves), catastrophe reserves and solvency requirements-though fitting different purposes and/or looked upon from different standpoints-always pose the same two problems for the actuary: the assessment of an appropriate overall reserve and the question of how to find the "right" distribution of the latter over a number of subportfolios or profit centers. For a solution of the second of these two 
problems put again

$$
\tilde{Y}=Y \quad \text { and } \quad \tilde{Z}=Z
$$

in the above mentioned general formula, thus

or

$$
\frac{E[Z]}{U} \cdot v[Z] \cdot\left(-\frac{\ln \varepsilon}{2}\right)=\delta
$$

$$
U=v[Z] \cdot\left(-\frac{\ln \varepsilon}{2}\right) \cdot \frac{1}{\delta} \cdot E[Z] .
$$

If now the entire portfolio is subdivided into a number of subportfolios $j=1$, $2, \ldots, N$ with totals of claims $Z_{j}$, we calculate $U_{j}$ according to

$$
U_{j}=v\left[Z_{j}\right] \cdot\left(-\frac{\ln \varepsilon^{\prime}}{2}\right) \cdot \frac{1}{\delta_{j}} E\left[Z_{j}\right]
$$

where $\varepsilon^{\prime}=$ constant, i.e., independent of $j$, which means that we consider a distribution of the total reserve over the subportfolios as fair if each subportfolio has the same ruin probability $\varepsilon^{\prime}$.

Of course $\sum_{j=1}^{N} U_{j}=U$, i.e.,

$$
\ln \varepsilon^{\prime} \cdot \sum_{j=1}^{N} v\left[Z_{j}\right] \frac{E\left[Z_{j}\right]}{\delta_{j}}=\ln \varepsilon \cdot v[Z] \frac{E[Z]}{\delta}
$$

which determines the common ruin probability $\varepsilon^{\prime}$.

$$
\ln \varepsilon^{\prime}=\ln \varepsilon \cdot \frac{\frac{\operatorname{Var}[Z]}{\delta E[Z]}}{\sum_{j=1}^{N} \frac{\operatorname{Var}\left[Z_{j}\right]}{\delta_{j} E\left[Z_{j}\right]}}
$$

Assuming non-correlated $Z_{j}$ thus yields

$$
\frac{-\ln \varepsilon^{\prime}}{-\ln \varepsilon}=\frac{\frac{\sum \operatorname{Var}\left[Z_{j}\right]}{\sum \delta_{j} E\left[Z_{j}\right]}}{\sum \frac{\operatorname{Var}\left[Z_{j}\right]}{\delta_{j} E\left[Z_{j}\right]}}
$$

where the right-hand side is always less than one because of

$$
\sum a_{j} b_{j}<\sum a_{j} \sum b_{j}
$$

since for nonnegative $a_{j}$ and $b_{j}$ one has $a_{j} b_{j}<a_{j} \sum b_{k}$ and by summing over $j$ one gets $\sum a_{j} b_{j}<\sum a_{j} \sum b_{k}$.

\section{4. “Extending” Scarce Statistical Materials}

A main difficulty to be overcome when assessing Cat Cover premiums is, for example, the fact that we only possess scarce statistical data as a rule. Instead 
of-or even better, parallel to-a parametric model approach it is sometimes useful to proceed pragmatically by combining underwriting judgement with scarce statistics as follows:

1st step: take the statistics available, say of the last five years, of claims exceeding the priority of the catastrophe fund.

2nd step: add the same five years statistics to it but with a built-in artificial windstorm claim which, we believe, is likely to occur every ten years.

3rd step: repeat the ten years statistics obtained in this way by building in an additional big fire and a catastrophe air crash.

4th step: repeat the above twenty years' statistics and add a severe windstorm with a return period of forty years.

5th step: the 40 years are again doubled and reinforced by a secular earthquake catastrophe.

\subsection{Quantifying the Change in IBNR}

Clearly if the organization is such that the component "change in IBNR" of the Earnings Statement is considered to be assessed by the actuary working in the Planning section to some extent, then there are a number of different methods at his disposition. Instead of describing them here even only sketchwise we refer to the excellent monograph "Loss Reserving Methods", Issue No 1 of Surveys of Actuarial Studies prepared by the Nationale-Nederlanden N.V., The Netherlands.

\subsection{Derivation of the Above Used Formula}

We merely indicate here the main steps of a proof of the general formula

$$
\frac{E[Z]}{U} \cdot v[Z] \cdot\left(-\frac{\ln \varepsilon}{2}\right)=\frac{E[\tilde{Y}] / E[Z]}{\operatorname{Var}[\tilde{Z}] / \operatorname{Var}[Z]}
$$

used before.

Cramér's inequality says that if $\varepsilon$ denotes the ruin probability and $\tilde{Y}$ the net result of the portfolio under consideration then

$$
\varepsilon \leqslant e^{-R U}
$$

with $U=$ initial reserve and $R=$ solution of $1=E\left[e^{-R \tilde{Y}}\right]$.

Putting equality in Cramér's inequality and taking logarithms on both sides yields

$$
-\frac{\ln \varepsilon}{U}=R
$$

where $R$ is the positive solution of $\varphi(R)=\ln E\left[e^{-R \tilde{Y}}\right]=0$. 
Taking only the first two terms of the expansion of $\varphi(R)$ we obtain

$$
-R E[\tilde{Y}]+\frac{R^{2}}{2} \operatorname{Var}[\tilde{Y}]=0 \quad \text { i.e. } R=\frac{2 E[\tilde{Y}]}{\operatorname{Var}[\tilde{Y}]}
$$

and therefore

$$
-\frac{\ln \varepsilon}{2 U}=\frac{E[\tilde{Y}]}{\operatorname{Var}[\tilde{Y}]}, \quad \text { where } \tilde{Y}=\text { net result }=\tilde{P}-\tilde{Z}
$$

Multiplying both sides by $\operatorname{Var}[Z]$ and dividing them by $E[Z]$ yields (realizing that $\operatorname{Var}[\tilde{Y}]=\operatorname{Var}[\tilde{Z}])$

$$
q=\frac{E[Z]}{U} \cdot v[Z] \cdot\left(-\frac{\ln \varepsilon}{2}\right)=\frac{E[\tilde{Y}] / E[Z]}{\operatorname{Var}[\tilde{Z}] / \operatorname{Var}[Z]}
$$

where the left-hand side (which we denote by $q=$ security factor) does not depend on the type of reinsurance (because no " " occurs), contrary to the right-hand side.

Interpretation of individual terms:

$$
\begin{aligned}
& \frac{U}{E[Z]}=\text { initial reserve in "natural" money units } E[Z] \\
& v[Z]=\frac{\operatorname{Var}[Z]}{E^{2}[Z]}=\text { square of the coefficient of variation of } Z \\
& \frac{E[\tilde{Y}]}{E[Z]}=\text { expected net result in natural money units } \\
& \frac{\operatorname{Var}[\tilde{Z}]}{\operatorname{Var}[Z]}=\text { some sort of reciprocal measure of the efficiency of } \\
& \text { the applied reinsurance programme. }
\end{aligned}
$$

In line with the intuition that the security factor decreases with increasing initial reserves, decreasing fluctuations of the gross result and increasing tolerated ruin probability (the latter being a measure of the risk aversion). 